\title{
SPECKLE IMAGING MEASUREMENTS OF THE RELATIVE TANGENTIAL VELOCITIES OF THE COMPONENTS OF T TAURI BINARY STARS
}

\author{
A. M. GHEZ ${ }^{1}$ \\ Department of Physics and Astronomy, University of California, Los Angeles, California 90095 \\ Electronic mail: ghez@urania.astro.ucla.edu

\section{A. J. Weinberger, G. Neugebauer, And K. Matthews} \\ California Institute of Technology 320-47, Pasadena, California 91125 \\ Electronic mail: alycia, gxn@mop.caltech.edu,kym@tacos.caltech.edu \\ D. W. MCCARTHY, JR. \\ Steward Observatory, University of Arizona, Tucson, Arizona 85271 \\ Electronic mail: mccarthy@as.arizona.edu \\ Received 1995 January 23; revised 1995 April 13
}

\begin{abstract}
Over a five year period, we have used speckle imaging to monitor $20 \mathrm{~T}$ Tauri binary stars with separations ranging from 0.09 to $1^{\prime \prime}(13-140 \mathrm{AU})$. This project is aimed at detecting the relative motion of the component stars to ascertain whether or not the observed companions (1) are stellar in nature, as opposed to being $\mathrm{HH}$ objects, and (2) are gravitationally bound to the primary stars. These observations demonstrate that speckle imaging measurements of close binary stars' separations can be made with an accuracy of a few milliarcseconds. The majority of the observed systems show significant relative velocities which (1) are not consistent with the motion expected for HH objects, (2) are greater than the velocity dispersion of these star-forming regions and thus are not the result of differential proper motion, and (3) are consistent with orbital motion. This is the first demonstration that these systems are physically bound. Furthermore, these relative velocity measurements provide dynamical evidence that the average total mass of these $\mathrm{T}$ Tauri binary star systems is $\sim 1.7 \mathscr{\ell}_{0}$. (C) 1995 American Astronomical Society.
\end{abstract}

\section{INTRODUCTION}

Recent imaging surveys have revealed the presence of a substantial population of $\mathrm{T}$ Tauri companion stars in Taurus and Ophiuchus with projected separations ranging from 1 to 150 AU (Ghez et al. 1993; Leinert et al. 1993; Simon et al. 1992, 1995). Unlike spectroscopic surveys, which have identified the very closest binary star systems (separations less than 1 AU; Mathieu et al. 1989), the imaging surveys obtain a single snapshot of the binary star pair in time and no dynamical information. It has been argued purely on a statistical basis that the imaged double stars are gravitationally bound. The binary star frequencies derived from these studies suggest that T Tauri stars are 2 to 4 times more likely to have a companion star in the separation range 1-150 $\mathrm{AU}$ than solar-type main sequence stars.

The discovery that $\mathrm{HV} \mathrm{Tau} / c$, a $K=11.6 \mathrm{mag}$ source separated by $5^{\prime \prime}$ from HV Tau (Simon et al. 1992), is a Herbig-Haro object as opposed to a stellar companion (Magazzu \& Martin 1994) raises the question of whether or not the high incidence of secondary sources near $\mathrm{T}$ Tauri stars can be explained by the presence of many more of these nonstellar $\mathrm{HH}$ sources. Herbig-Haro objects are thought to be shock excitation from the interaction of high-velocity stellar winds with ambient molecular gas (cf. reviews by Schwartz 1983; Edwards et al. 1993). These shocks are not stationary and have been observed to move at significant fractions of

${ }^{1}$ Hubble Fellow. the jet's speed (cf. Schwartz 1986). With respect to a T Tauri star these sources are expected to move out radially at speeds of $\sim 100 \mathrm{~km} / \mathrm{s}$. At the distance to Taurus and Ophiuchus, 140 pc (Kenyon et al. 1994; DeGeus \& Burton 1991), the relative separation between a Herbig-Haro source and a T Tauri star should increase at a rate of $\sim 150$ milliarcsecond (mas)/yr. This should easily be detected with speckle imaging, which typically can measure binary source separations to within a few milliarcseconds. This can be compared to that expected for two gravitationally bound binary stars with semimajor axes ranging from 13 to $140 \mathrm{AU}$. In the case of orbital motion the velocities are expected to be (1) an order of magnitude smaller and (2) should depend on the average separation of the binary star.

If the secondary sources are gravitationally bound, they can physically influence the formation and evolution of a circumstellar disk, if such a disk is present, in a number of ways. Secondary stars at distances less than 100 AU are expected to have a disruptive influence on a circumstellar disk. This is supported by (1) observations of lower disk masses for close binary star systems compared to single or wide (>100 AU) binary star systems (Jensen et al. 1994; Osterloh \& Beckwith 1995; Simon et al. 1995) and (2) evidence of disk clearing due to a close companion star in the individual cases of GG Tau (Dutrey et al. 1994), UZ Tau (Ghez et al. 1994), and GW Ori (Mathieu et al. 1991). Furthermore, secondary stars in systems with larger semimajor axes, but in eccentric orbits such that they are occasionally within the circumstellar disk, are thought to be able to trigger acceler- 
ated accretion events known as FU Ori outbursts (Bonnell \& Bastien 1992). Even secondaries that never enter the circumstellar disk are expected to tidally induce somewhat accelerated accretion (Ostriker et al. 1992) or possibly effect the geometry of the disk (Terquem \& Bertout 1993). Rapid erosion of a circumstellar disk as a result of these interactions could seriously compromise the potential for planetary formation in such close binary star systems.

If the secondaries are both stellar and bound, the prevalence of binary star systems at the T Tauri star stage of evolution, roughly $2-3 \times 10^{6} \mathrm{yr}$ (Simon et al. 1993), suggests that star-formation processes produce primarily multiple star systems. Among the multiple star-formation mechanisms being studied today are core fragmentation (e.g., Boss 1993; Bonnell \& Bastein 1993), disk fragmentation (e.g., Adams et al. 1989; Bonnell 1994), and capture in small clusters following prompt initial fragmentation (McDonald \& Clarke 1993,1995). It is only through detailed observational studies of binary star systems that we will be able to derive the masses of the binary star components and thereby constrain these theoretical binary star-formation models.

In this study the relative separations of a number of close $\mathrm{T}$ Tauri binary stars in Taurus and Ophiuchus are monitored using speckle imaging. At the distance to both of these starforming regions, the initial angular separations of the systems studied, 0.09 to $1^{\prime \prime}$, correspond to a projected linear separation range of 13-140 AU. The main objective of this work is to determine whether or not these systems are indeed (1) stellar and (2) gravitationally bound and consequently actively perturbing their local environments. An additional aim of this program is to obtain dynamical estimates of the bound systems' component masses, which would not only constrain possible binary star formation mechanisms, but would also provide fundamental tests of evolutionary tracks.

\section{OBSERVATIONS AND DATA ANALYSIS}

The near-infrared speckle imaging program aimed at monitoring $\mathrm{T}$ Tauri binary star separations was an outgrowth of the multiplicity study described in Ghez et al. (1993). Initially this program was conducted when the seeing conditions were not sufficiently good observe the fainter objects in the multiplicity survey and thus the targets were those binary stars detected by Ghez et al. which were brighter than $K \leqslant 7.5 \mathrm{mag}$. Since the completion of the multiplicity work in Taurus and Ophiuchus (1992 February) the source list was extended to all binary stars in Ghez et al. This program also included observations of a number of binary stars with known orbital elements, selected from Worley \& Heintz (1983; Grade $\leqslant 3$ ), to calibrate the pixel scales and orientations of the speckle cameras.

The observations were carried out in a number of (typically six) of object/reference "pairs." A pair consists of a series of 400 to 500 snapshots (100 ms exposures) on the object of interest adjacent in time with a similar series of measurements on a reference source. For these observations a reference star is one that is unresolved out to the diffraction limit of the telescope. Several reference stars were generally used for the initial measurements and the optimal one, i.e., one that is truly unresolved, bright, and closest to the source, was used for subsequent observations (see Table 2 of Ghez et al. 1993). In addition, a series of snapshots on blank sky were obtained at the beginning and end of each sequence of object/reference pairs.

Between 1989 December and 1992 February, measurements were obtained at the $f / 415$ Cassegrain focus of the Hale $5 \mathrm{~m}$ telescope of Palomar Observatory using a $58 \times 62$ InSb array camera, a system we shall refer to as CIT-1a. The initial observations of $\mathrm{T}$ Tauri binary stars included in this program were previously reported in Ghez et al. 1993. In 1993 May this system was modified with reimaging optics just outside the dewar such that the standard $f / 70$ Cassegrain secondary could be used (CIT-1b). This offered the advantage of two interchangeable pixel scales, a large scale for direct imaging and a fine scale for speckle imaging. Measurements were made with this system between 1993 May and 1993 November. This system was modified again in 1994 October to incorporate a larger format and lower readnoise array $(256 \times 256 \mathrm{InSb}$; CIT-2): the new reimaging optics were used to obtain a pixel scale that Nyquist sampled the field of view for speckle imaging. Additional observations from 1992 October to 1994 September were obtained at the Steward Observatory $2.3 \mathrm{~m}$ with a two-dimensional speckle imaging camera similar to CIT-1a and b (cf. McCarthy et al. 1990; SO-1). This camera has four possible pixel scales, but only one was used for the observations presented here. Measurements by all cameras were generally made in the photometric $K(2.2 \mu \mathrm{m})$ band, with the exception of SO-1 observations of the closest binary stars $(\leqslant 0$ "1), which were obtained in the $H(1.65 \mu \mathrm{m})$ band, such that these systems could be spatially resolved with the $2.3 \mathrm{~m}$ telescope. Table 1 summarizes the properties of the cameras used.

The procedure for recovering a diffraction-limited image of an object, or equivalently the object's Fourier amplitudes and phases, from each obj/ref pair has been described in detail elsewhere (cf. Ghez 1992; Koresko et al. 1991; McCarthy et al. 1991) and thus here we will only describe how a binary star's parameters are obtained from these end products of speckle imaging. A binary star is revealed in the final image as two delta functions and in the power spectrum (the square of the object's Fourier amplitudes) as a sinusoid,

$$
P(\mathbf{f})=\frac{R^{2}+1+2 R \cos (2 \pi \mathbf{f s})}{R^{2}+1+2 R},
$$

where $R$ is the binary star's flux ratio and $\mathbf{s}$ is its vector separation on the sky. Since systematics due to both detector readout electronics and miscalibration of the atmosphere are easier to identify and isolate in the Fourier domain than the image domain (cf. Ghez et al. 1993), the binary stars' vector separations and flux ratios are estimated through twodimensional fits of the function given in Eq. (1) to a weighted average of the power spectra produced from the obj/ref pairs. The uncertainties reported here are the standard deviations of the binary star's parameters derived from the individual obj/cal pair's power spectra. Fits to the power spectra from each obj/ref pair, as opposed to their average, demonstrate that the formal uncertainties from the minimization of chi-squared occasionally underestimate the actual un- 
TABLE 1. Speckle cameras.

\begin{tabular}{|c|c|c|c|c|c|}
\hline \multirow[t]{2}{*}{ Dates } & \multirow[t]{2}{*}{ Camera } & \multicolumn{2}{|c|}{ Average } & \multicolumn{2}{|c|}{ Relative to CIT-1a } \\
\hline & & $\begin{array}{l}\text { Pixel Scale } \\
\text { (mas/pix) }\end{array}$ & $\begin{array}{l}\text { Orientation } \\
\qquad(\text { deg })\end{array}$ & Pixel Scale & $\begin{array}{l}\text { Orientation } \\
\qquad(\text { deg })\end{array}$ \\
\hline $1989 \mathrm{Dec}-1992 \mathrm{Feb}$ & CIT-1a & $53( \pm 1)$ & $0( \pm 1)$ & - & - \\
\hline 1993 May - 1993 Nov & CIT-1b & $38.7( \pm 0.9)$ & $-0.9( \pm 0.6)$ & $0.726( \pm 0.004)$ & $0.0( \pm 0.2)$ \\
\hline 1994 Oct - 1994 Dec & CIT-2 & $35.8( \pm 0.9)$ & $-4.0( \pm 1)$. & $0.665( \pm 0.005)$ & $-0.3( \pm 0.4)$ \\
\hline \multirow[t]{2}{*}{1992 Oct - 1994 Sept } & SO-1 & $70( \pm 2)$. & $5.7( \pm 1.4)$ & $1.30( \pm 0.02)$ & $6( \pm 1)$ \\
\hline & SO-1' & - & - & $2.62 \times 10^{-3} \phi_{2}+1.198( \pm 0.007)$ & $-0.122 \phi_{1}+13.1( \pm 0.3)$ \\
\hline
\end{tabular}

Notes: (1) The pixel scales and orientations used in this paper tie all the measurements to CIT-1a. Thus the pixel scales and orien-

tations for CIT-1b, CIT-2, SO-1, and SO-1' (SO-1 corrected for field distortion) are based on the relative scales/orientations and the average estimate of the scale/orientation of CIT-1a. (2) $\phi_{1}$ and $\phi_{2}$ range from 0 to $90^{\circ-}$ and are the angles from the N-S and the NE-SW axes respectively of SO-1.

certainties in the derived quantities. Solutions based on the power spectrum alone leave a $180^{\circ}$ ambiguity in the binary star's position angle. This is resolved by examining the final image, or equivalently the object's Fourier phases.

It should be noted that the approach taken here differs slightly from that in Ghez et al. (1993), where the fits are made to projected one-dimensional power spectra and the uncertainties are based on the formal fits. Nonetheless, for the observations reported both in Ghez et al. and here, the derived binary star parameters are consistent given the current uncertainties.

Two of the four measurements of SR20, the most closely separated binary star in the sample, require special mention. In both the 1990 and 1993 observations the binary star is detected and measured, as described above, to have the same flux ratio but a different separation in 1990 than in 1993. In 1991 and 1992 the companion star was not detectable due to poorer seeing conditions. Therefore for these years limits for the binary star separation were determined from the power spectra as outlined in Ghez et al. (1993) assuming that the flux ratio was constant as suggested by the 1990 and 1993 measurements.

\section{RESULTS}

The accuracy to which the power spectra can be fit to the model, and hence the uncertainties in a binary star's parameters, is affected by several factors (1) the brightness of the source, (2) the binary star's separation and flux ratio, (3) the maximum spatial frequency measured, (4) the value and stability of the atmospheric coherence length and time, and (5) how well the detector's pixels have been mapped onto the sky. In general, the uncertainties in the vector separation measured with respect to the detector for both the program and calibration binary stars average $\sim 1 \%$. Mapping these separations onto the sky contributes an additional $2 \%$ uncertainty as described in the Appendix.
Table 2 lists the measurements of the T Tauri binary stars' vector separations with respect to the sky. Although a pixel scale of 53 mas/pixel for CIT-1a is assumed in these measurements, the uncertainties in this absolute scale (2\%), which would be added in quadrature, have not been incorporated. The reported uncertainties are from the fitting procedure and the relative pixel scale and orientation determinations alone (see the Appendix). In this manner, it is possible to demonstrate that a change in separation has occurred without introducing the additional uncertainty of the absolute pixel scales. Figure 1 plots the relative separation measurements. These points can also be viewed as the relative position of the secondary stars assuming that the primary stars are located at the origin $(0,0)$ of each plot. It is clear that many of the binary star separations have changed over time.

The sensitivity to separation changes increases as the time baseline of the measurements increases. Since observations made by other groups of these subarcsec $\mathrm{T}$ Tauri binary stars have generally been made only within the last few years and the relative pixel scales are poorly known, any differences between those measurements and with the measurements presented here are dominated by uncertainties in the relative pixel scales and do not increase the sensitivity to the relative motion of the binary star components. If, however, the measurements are old enough, the change in separation can exceed the uncertainties in the relative pixel scales. This is the case for 162218-2420 (ADS 10042), whose separation was measured by micrometer in both 1922 (Aitken 1932; separation $=0.22$ and P.A. $=205.6$ ) and 1974 (Heinz 1980; separation $=0.23$ and P.A. $=163^{\circ} 6$ ). Although the uncertainties in these measurements are larger than those of the current measurements, significant relative motions are observable over this time baseline. Therefore we include these two measurements in our calculations. To assess the significance of the observed separation change for each binary star pair, linear fits were made to the relative separation versus time in both Cartesian and polar coordinates. Figure 2 displays the 
TABLE 2. (a) T Tauri binary star parameters (Taurus).

\begin{tabular}{|c|c|c|c|c|}
\hline Object & Date & Camera & $\begin{array}{c}\text { Separation }^{\mathrm{a}} \\
\text { (arcsec) }\end{array}$ & $\begin{array}{l}\mathrm{PA}^{\mathrm{a}} \\
(\text { deg })\end{array}$ \\
\hline \multirow[t]{5}{*}{ DF Tau } & 1990 Nov 09 & CIT-1a & $0.090 \pm 0.002$ & $328 \pm 3$ \\
\hline & 1992 Oct 10 & SO-1 & $0.098 \pm 0.008$ & $312 \pm 5$ \\
\hline & 1993 Nov 25 & CIT-1b & $0.096 \pm 0.004$ & $309.5 \pm 0.7$ \\
\hline & 1994 Oct 18 & CIT-2 & $0.089 \pm 0.002$ & $302 \pm 3$ \\
\hline & 1994 Dec 19 & CIT-2 & $0.089 \pm 0.001$ & $301 \pm 1$ \\
\hline \multirow[t]{4}{*}{ V773 Tau } & 1990 Oct 03 & CIT-1a & $0.1118 \pm 0.0005$ & $294.1 \pm 0.5$ \\
\hline & 1992 Oct 10 & SO-1 & $0.12 \pm 0.02$ & $307 \pm 5$ \\
\hline & 1993 Nov 25 & CIT-1b & $0.092 \pm 0.008$ & $304 \pm 8$ \\
\hline & 1994 Oct 19 & CIT-2 & $0.0656 \pm 0.0006$ & $318 \pm 2$ \\
\hline \multirow[t]{2}{*}{ V410 Tau } & 1991 Oct 20 & CIT-1a & $0.13 \pm 0.01$ & $183 \pm 5$ \\
\hline & 1994 Oct 20 & CIT-2 & $0.074 \pm 0.002$ & $182 \pm 5$ \\
\hline \multirow[t]{3}{*}{ FO Tau } & 1991 Oct 18 & CIT-1a & $0.161 \pm 0.001$ & $181.7 \pm 0.6$ \\
\hline & 1994 Oct 20 & CIT-2 & $0.153 \pm 0.002$ & $190.6 \pm 0.4$ \\
\hline & 1994 Dec 19 & CIT-2 & $0.154 \pm 0.002$ & $191.2 \pm 0.4$ \\
\hline \multirow[t]{2}{*}{ V928 Tau } & 1991 Oct 19 & CIT-1a & $0.163 \pm 0.005$ & $124.1 \pm 0.5$ \\
\hline & 1994 Dec 19 & CIT-2 & $0.181 \pm 0.002$ & $121.2 \pm 0.6$ \\
\hline \multirow[t]{3}{*}{$\mathrm{LkH} \alpha 332 / \mathrm{G} 1$} & 1990 Oct 04 & CIT-1a & $0.208 \pm 0.002$ & $257.6 \pm 0.4$ \\
\hline & 1993 Nov 25 & CIT-1b & $0.23 \pm 0.01$ & $263 \pm 2$ \\
\hline & 1994 Oct 18 & CIT-2 & $0.224 \pm 0.002$ & $262.7 \pm 0.4$ \\
\hline \multirow[t]{3}{*}{ GG Tau } & 1991 Oct 21 & CIT-1a & $0.26 \pm 0.01$ & $2 \pm 1$ \\
\hline & 1994 Sep 24 & SO-1 & $0.258 \pm 0.004$ & $357 \pm 2$ \\
\hline & 1994 Oct 18 & CIT-2 & $0.242 \pm 0.003$ & $0.9 \pm 0.5$ \\
\hline \multirow[t]{2}{*}{$\mathrm{XZ}$ Tau } & 1991 Oct 20 & CIT-1a & $0.31 \pm 0.01$ & $151 \pm 2$ \\
\hline & 1994 Dec 19 & CIT-2 & $0.296 \pm 0.002$ & $147.0 \pm 0.4$ \\
\hline \multirow[t]{2}{*}{ GH Tau } & 1991 Oct 19 & CIT-1a & $0.314 \pm 0.001$ & $298.4 \pm 0.1$ \\
\hline & 1994 Dec 19 & CIT-2 & $0.307 \pm 0.002$ & $296.0 \pm 0.4$ \\
\hline \multirow[t]{2}{*}{ UZ Tau W } & 1990 Nov 10 & CIT-1a & $0.359 \pm 0.002$ & $357.4 \pm 0.3$ \\
\hline & 1994 Dec 19 & CIT-2 & $0.360 \pm 0.003$ & $1.3 \pm 0.4$ \\
\hline \multirow[t]{4}{*}{ V807 Tau } & 1990 Nov 10 & CIT-1a & $0.3710 \pm 0.0005$ & $332.5 \pm 0.2$ \\
\hline & 1993 Nov 26 & CIT-1b & $0.34 \pm 0.02$ & $334 \pm 6$ \\
\hline & 1994 Sep 24 & SO-1 & $0.336 \pm 0.003$ & $326.9 \pm 0.9$ \\
\hline & 1994 Oct 18 & CIT-2 & $0.329 \pm 0.004$ & $328.1 \pm 0.6$ \\
\hline
\end{tabular}


TABLE 2. (a) (continued)

\begin{tabular}{|c|c|c|c|c|}
\hline Object & Date & Camera & $\begin{array}{c}\text { Separation }^{\mathrm{a}} \\
\text { (arcsec) }\end{array}$ & $\begin{array}{l}\mathrm{PA}^{\mathrm{a}} \\
(\text { deg })\end{array}$ \\
\hline \multirow[t]{9}{*}{ T Tau } & $1989 \operatorname{Dec} 10$ & CIT-1a & $0.720 \pm 0.004$ & $175.5 \pm 0.2$ \\
\hline & 1990 Nov 10 & CIT-1a & $0.7155 \pm 0.0005$ & $176.15 \pm 0.02$ \\
\hline & 1991 Nov 19 & CIT-1a & $0.702 \pm 0.003$ & $176.41 \pm 0.05$ \\
\hline & 1992 Feb 20 & CIT-1a & $0.710 \pm 0.004$ & $176.69 \pm 0.09$ \\
\hline & 1992 Oct 11 & SO-1 & $0.692 \pm 0.008$ & $177 \pm 1$ \\
\hline & 1993 Nov 25 & CIT-1b & $0.701 \pm 0.004$ & $177.0 \pm 0.2$ \\
\hline & 1993 Dec 26 & SO-1 & $0.690 \pm 0.005$ & $176.0 \pm 0.5$ \\
\hline & 1994 Sep 22 & SO-1 & $0.689 \pm 0.005$ & $175.9 \pm 0.6$ \\
\hline & 1994 Oct 19 & CIT-2 & $0.689 \pm 0.006$ & $178.2 \pm 0.4$ \\
\hline \multirow[t]{4}{*}{ UY Aur } & 1990 Nov 10 & CIT-1a & $0.8750 \pm 0.0005$ & $227.1 \pm 0.1$ \\
\hline & 1993 Dec 26 & SO-1 & $0.861 \pm 0.008$ & $227.6 \pm 0.4$ \\
\hline & 1994 Sep 22 & SO-1 & $0.868 \pm 0.005$ & $226.5 \pm 0.4$ \\
\hline & 1994 Oct 18 & CIT-2 & $0.887 \pm 0.008$ & $228.2 \pm 0.3$ \\
\hline
\end{tabular}

(a) The reported uncertainties in separation and position angle are from the fit and relative pixel scale/orientation determinations.

The additional uncertainties of the absolute pixel scale/orientation of CIT-1a (see Table 1), which would be added in quadrature,

have not been included.

measurements and fits in Cartesian coordinates (relative R.A. separation versus time and relative DEC separation versus time) for three binary stars which have been measured during at least five separate observing runs: DF Tau, 160946-1851, and $\mathrm{T}$ Tau. These examples show that the observed separations as a function of time over the baseline of this study, which is relatively short compared to the anticipated orbital periods, are well represented by a straight line. The resultant velocities and their uncertainties are shown in Fig. 3. Seventeen of the observed T Tauri binary stars have measured relative velocities that are $2 \sigma$ greater than zero and thus appear to have moved significantly (see Table 3). The maximum relative separation change measured is $\sim 13 \mathrm{~km} / \mathrm{s}$ (SR 20 and V410 Tau) and the average is $5 \mathrm{~km} / \mathrm{s}$, where the distance assumed for each star is given in column 2 of Table 3 .

\section{DISCUSSION}

\subsection{The Origin of the Observed Relative Motion}

The relative motion of a T Tauri star with respect to (1) a Herbig-Haro object, (2) a background source, (3) another cloud member, and (4) a gravitationally bound object are distinctly different. Thus the nature of the secondary sources can be identified on the basis of the relative velocity measurements, plotted in Fig. 3. The possibility that any of these sources are Herbig-Haro objects is ruled out for a number of reasons. First, a Herbig-Haro object is expected to move along the direction of the star's wind, which requires the direction of the relative motion between a Herbig-Haro ob- ject and a T Tauri star to be purely radial and positive $\left(v_{\theta}=0\right.$; $\left.v_{r}>0\right)$. The radial and azimuthal components of the relative velocities [Fig. 3(b)] reveal that most of the sources move significantly in the azimuthal direction. Furthermore only one source's motion is consistent with being purely radial and positive, AS205. A second reason to believe that the secondary sources are not Herbig-Haro objects is the magnitude of the relative velocities. On average the binary star separations are changing at a rate of $5 \mathrm{~km} / \mathrm{s}$. Furthermore the components of the only source whose relative velocity direction is radial and positive has a relative speed of only $2 \mathrm{~km} / \mathrm{s}$. This is two orders of magnitude smaller than that expected for the relative motion of a Herbig-Haro object with respect to a T Tauri star, $\sim 100 \mathrm{~km} / \mathrm{s}$. Thus we conclude that none of the secondary sources in this study are Herbig-Haro objects.

These measurements also rule out the possibility that the nearby companions are the result of chance superpositions with either background sources or other members of the cloud. If a secondary source is a background object then it would appear as a fixed object and the relative motion between it and the $T$ Tauri star would be equal to the absolute proper motion of the T Tauri star on the sky, +180 or $0^{\circ}$ depending on which component is the optically identified $\mathrm{T}$ Tauri star. The mean proper motion of $\mathrm{T}$ Tauri stars in Taurus is $23 \mathrm{mas} / \mathrm{yr}$ (Jones \& Herbig 1979), which corresponds to $15 \mathrm{~km} / \mathrm{s}$ at a distance of $140 \mathrm{pc}$, and in Oph-Sco is 37 mas/yr (Petrie 1961), which corresponds to 25 and $28 \mathrm{~km} / \mathrm{s}$ at 140 and $160 \mathrm{pc}$, respectively. Since the relative motions are significantly smaller than the proper motion of their re- 
TABLE 2. (b) T Tauri binary star parameters (Oph-Sco).

\begin{tabular}{|c|c|c|c|c|}
\hline Object & Date & Camera & $\begin{array}{c}\text { Separation }^{a} \\
\text { (arcsec) }\end{array}$ & $\begin{array}{l}\mathrm{PA}^{\mathrm{a}} \\
(\text { deg })\end{array}$ \\
\hline \multirow[t]{4}{*}{ SR 20} & 1990 July 09 & CIT-1a & $0.07 \pm 0.01$ & $227 \pm 5$ \\
\hline & 1991 May 04 & CIT-1a & $<0.069$ & - \\
\hline & 1992 July 23 & CIT-1a & $<0.065$ & - \\
\hline & 1993 July 02 & CIT-1b & $0.038 \pm 0.001$ & $177 \pm 9$ \\
\hline \multirow[t]{4}{*}{ ROXs $42 \mathrm{C}$} & 1990 July 08 & CIT-1a & $0.15 \pm 0.02$ & $136 \pm 5$ \\
\hline & 1991 May 03 & CIT-1a & $0.155 \pm 0.003$ & $140.1 \pm 0.9$ \\
\hline & 1992 July 24 & CIT-1a & $0.173 \pm 0.002$ & $141.1 \pm 0.5$ \\
\hline & 1993 July 02 & CIT-1b & $0.185 \pm 0.004$ & $142.4 \pm 0.7$ \\
\hline \multirow[t]{5}{*}{$160946-1851$} & 1990 Aug 05 & CIT-1a & $0.208 \pm 0.002$ & $160.4 \pm 0.6$ \\
\hline & 1991 May 04 & CIT-1a & $0.212 \pm 0.005$ & $162 \pm 3$ \\
\hline & 1992 May 12 & CIT-1a & $0.21 \pm 0.02$ & $160 \pm 4$ \\
\hline & 1992 July 25 & CIT-1a & $0.212 \pm 0.005$ & $158.6 \pm 0.5$ \\
\hline & 1993 July 01 & CIT-1b & $0.209 \pm 0.002$ & $159 \pm 1$ \\
\hline \multirow[t]{2}{*}{$162218-2420$} & 1990 July 8 & CIT-1a & $0.247 \pm 0.005$ & $157.5 \pm 0.9$ \\
\hline & 1992 July 25 & CIT-1a & $0.234 \pm 0.004$ & $155.2 \pm 0.6$ \\
\hline \multirow[t]{2}{*}{$155913-2233$} & 1990 July 8 & CIT-1a & $0.283 \pm 0.004$ & $347.7 \pm 0.7$ \\
\hline & 1993 July 04 & CIT-1b & $0.292 \pm 0.008$ & $345 \pm 2$ \\
\hline \multirow[t]{6}{*}{ SR 9} & 1990 July 09 & CIT-1a & $0.580 \pm 0.002$ & $349.7 \pm 0.4$ \\
\hline & 1990 Aug 05 & CIT-1a & $0.580 \pm 0.002$ & $349.8 \pm 0.4$ \\
\hline & 1991 May 03 & CIT-1a & $0.583 \pm 0.005$ & $349.7 \pm 0.2$ \\
\hline & 1992 Feb 18 & CIT-1a & $0.58 \pm 0.01$ & $350.5 \pm 0.1$ \\
\hline & 1992 July 25 & CIT-1a & $0.588 \pm 0.003$ & $350.5 \pm 0.1$ \\
\hline & 1993 July 02 & CIT-1b & $0.592 \pm 0.004$ & $350.0 \pm 0.2$ \\
\hline \multirow[t]{2}{*}{ AS 205} & 1990 July 7 & CIT-1a & $1.313 \pm 0.003$ & $212.0 \pm 0.2$ \\
\hline & 1992 May 13 & CIT-1a & $1.317 \pm 0.002$ & $211.87 \pm 0.08$ \\
\hline
\end{tabular}

(a) see footnote to Table $2 \mathrm{a}$

spective association or in the wrong direction in the case of V410 Tau, we conclude that none of the secondary sources in this sample are background stars.

In contrast to the large relative velocity expected from the motion of A T Tauri star relative to a distance background source, the anticipated rate of separation change for a double star created by the chance superposition of two unassociated T Tauri stars in the cloud is quite small. In this case the relative motion is caused by the differential between the proper motions of the two $\mathrm{T}$ Tauri stars and thus on average should have a magnitude equal to the velocity dispersion of the star forming regions studied, which is $\sim 2 \mathrm{~km} / \mathrm{s}$ for Tau- rus (Jones \& Herbig 1979) and $\sim 1 \mathrm{~km} / \mathrm{s}$ for Oph-Sco (Bertian 1958). In Fig. 3 circles of radii 1 and $2 \mathrm{~km} / \mathrm{s}$, centered at zero velocity, are drawn to show that the measured relative velocity is generally larger than the clouds' dispersion for all but four sources: AS 205, 155913-2233, 160946-1851, and UY Aur. This leads us to the conclusion that the majority of sources in this sample must be gravitationally bound. The others may also be physically associated binary stars, but it is not possible to ascertain this from the magnitude of the relative velocities alone.

To verify the origin of the observed motions, we examined the relative velocities of each pair versus their mean 

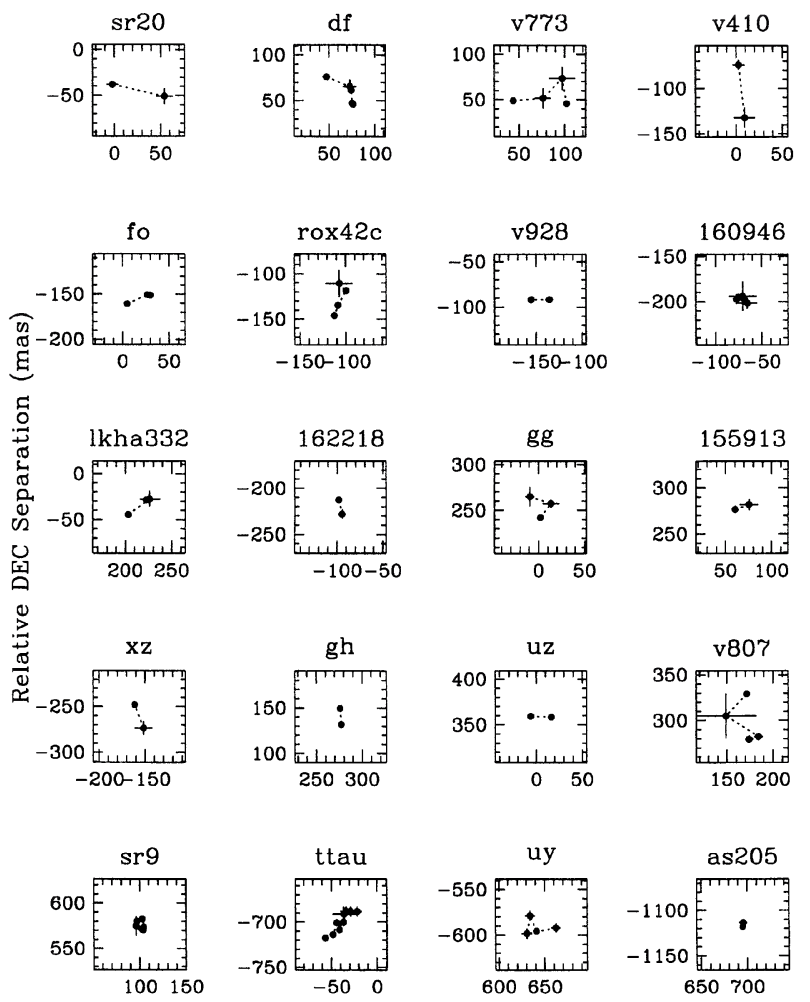

Relative RA Separation (mas)

FIG. 1. Each panel $(100 \times 100$ mas $)$ contains the relative separation measurements for a given $\mathrm{T}$ Tauri binary star. Each point represents an independent measurement of the binary star's vector separation and the dashed lines connect these measurements sequentially in time. Points with no error bars shown have uncertainties which are smaller than the size of the points. These plots show that the majority of the observed $T$ Tauri binary stars have moved significantly from their initial relative positions.

separations. If the relative motion is a consequence of orbital motion, the relative velocities should be greater for those systems with smaller separations. This can easily be demonstrated: the orbital period, $P$ (in years), of a binary star equals

$$
P^{2}=\frac{A^{3}}{\mathscr{C b}_{\text {tot }}},
$$

where $A$ is the semimajor axis in units of $\mathrm{AU}$, and $\mathscr{C}_{\text {tot }}$ is the total mass of the system in units $\mathscr{C}_{0}$. In the case of a face-on circular orbit the relative velocity, $v_{\text {rel }}$ (in AU/yr), of the two components equals

$$
v_{\mathrm{rel}}=2 \pi \sqrt{\frac{\mathscr{M}_{\mathrm{tot}}}{A}} \text {. }
$$

In general $v_{\text {rel }}$ also depends on the orbit's inclination and eccentricity, but nonetheless the overall trend for the relative velocities of components in bound systems to increase with decreasing separations should still hold.

Figure 4 shows the relative velocity versus the mean separation for all the binary stars in the sample with the exception of V807 Tau whose motion over the course of this study is most likely dominated by a third companion (see Sec. 4.2). The pairs with the smallest separations are experiencing the
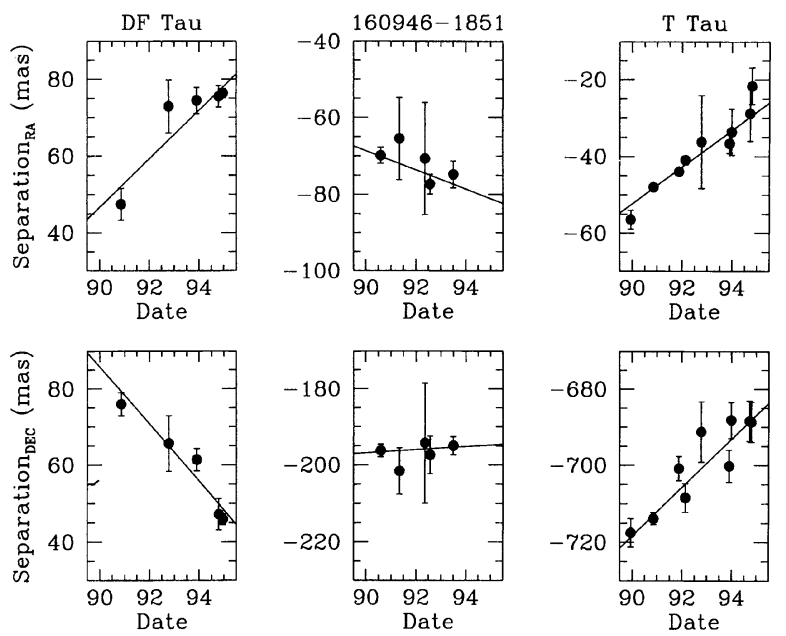

FIG. 2. The relative R.A. separation vs time and the relative DEC separation vs time measurements are shown in the top and bottom rows, respectively, for the binary stars DF Tau, 160946-1851, and T Tau. The uncertainties are the standard deviations of the separations derived for the individual obj/ref pairs (described in Sec. 2). These plots show that over the time baseline of this study, which is relatively short compared to the anticipated orbital periods, the data can be fit to a straight line. For each binary stars this provides a measure of the relative velocity between its two components.

largest separation changes. The average relative velocity measured for binary stars with projected separations less than $35 \mathrm{AU}$ (the "close" binary stars set) is $7 \pm 1 \mathrm{~km} / \mathrm{s}$ whereas for those separated by more than $35 \mathrm{AU}$ (the "wide" binary star set) is $3.5 \pm 0.5 \mathrm{~km} / \mathrm{s}$. If the observed relative velocities are a consequence of orbital motion, then they should also be related to the total mass $\left(\mathscr{C}_{\text {tot }}\right)$ of the binary star systems. Although it is not presently possible to estimate the mass of the secondary star $\left(\mathscr{C l}_{2}\right)$ in the close T Tauri stars, the primary star's mass $\left(\mathscr{C}_{1}\right)$ can be estimated from the measured spectral type, the previous determinations of the stellar luminosity, the observed flux ratio, and theoretical tracks as was done in Ghez et al. (1993; see their Table 7). In Fig. 4 the binary stars with $\mathscr{M}_{1}>1 \mathscr{C}_{0}$ are plotted as squares and the systems with $\mathscr{C}_{1} \leqslant 1 \mathscr{C}_{0}$ as circles. Table 4 divides the sample by separation and $\mathscr{C}_{1}$. Most of the high $\mathscr{C}_{1}$ stars are in the close binary star set and within this set the stars with $\mathscr{U}_{1}>\mathscr{C l}_{0}$ are moving faster than those with $\mathscr{M b}_{1}<1 \mathscr{C l}_{0}$. The correlation with both separation and primary star mass supports our earlier assertion that the observed motion is the result of orbital motion. This is the first demonstration that these systems are physically bound.

Since the position angles of these systems have changed at most by $50^{\circ}$ in the case of SR20 and on average by $8^{\circ}$ $\pm 3^{\circ}\left(17^{\circ} \pm 7^{\circ}\right.$ for the close set and $3.2^{\circ} \pm 0.5^{\circ}$ for the wide set), it is apparent that these binary stars have generally traversed only a small fraction of their orbits over the course of this study. Thus orbital element solutions and therefore masses are poorly determined at this point. It is however instructive to compare the measured velocities and separations with that expected for a sample of binary stars randomly observed. To construct the comparison curves, we ran a number of computer simulations similar to those described by Fischer \& Marcy (1992) to calculate the relationship be- 


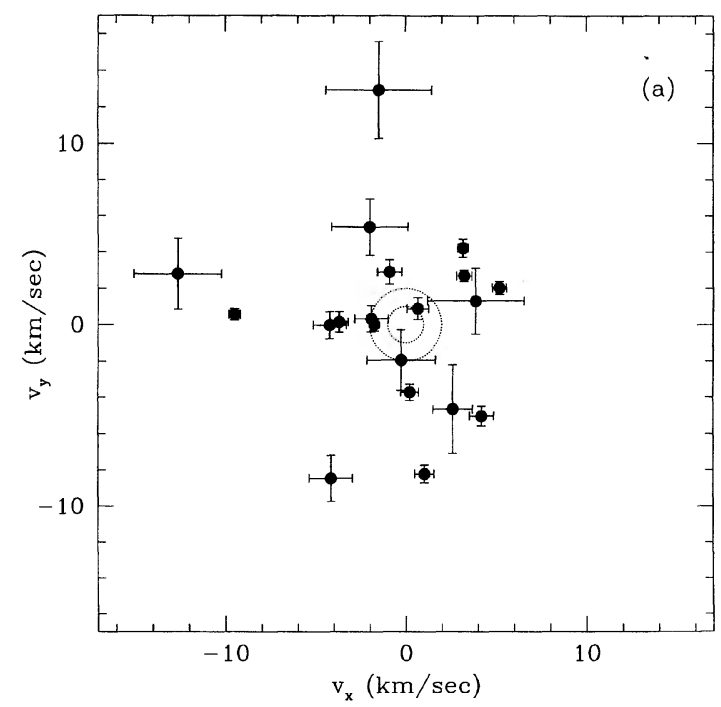

TABLE 3. Relative velocities.

\begin{tabular}{|c|c|c|c|}
\hline Name & $\begin{array}{c}\text { Distance } \\
\qquad(p c)\end{array}$ & $\begin{array}{l}\text { Avg. Sepn. } \\
\qquad(A U)\end{array}$ & $\begin{array}{c}\mathrm{V}_{\mathrm{tot}} \\
(\mathrm{km} / \mathrm{sec})\end{array}$ \\
\hline DF Tau & 140 & 12.9 & $6.6 \pm 0.6$ \\
\hline V773 Tau & 140 & 13.7 & $9.5 \pm 0.3$ \\
\hline V410 Tau & 140 & 14.5 & $13 \pm 3$ \\
\hline FO Tau & 140 & 21.8 & $5.6 \pm 0.4$ \\
\hline V928 Tau & 140 & 24.1 & $4.2 \pm 0.9$ \\
\hline $\mathrm{LkH} \alpha 332 / \mathrm{G} 1$ & 140 & 30.8 & $4.2 \pm 0.4$ \\
\hline GG Tau & 140 & 35.7 & $5 \pm 2$ \\
\hline XZ Tau & 140 & 42.6 & $6 \pm 2$ \\
\hline GH Tau & 140 & 43.5 & $3.7 \pm 0.5$ \\
\hline UZ Tau W & 140 & 50.3 & $3.7 \pm 0.5$ \\
\hline V807 Tau & 140 & 48.1 & $8.3 \pm 0.5$ \\
\hline T Tau & 140 & 98.1 & $5.3 \pm 0.5$ \\
\hline UY Aur & 140 & 122.2 & $1.1 \pm 0.6$ \\
\hline SR 20 & 140 & 7.9 & $13 \pm 2$ \\
\hline ROX 42C & 140 & 23.3 & $9 \pm 1$ \\
\hline $160946-1851$ & 160 & 32.6 & $1.9 \pm 0.9$ \\
\hline $162218-2420$ & 140 & 33.7 & $1.7 \pm 0.2$ \\
\hline $155913-2233$ & 160 & 46.0 & $4 \pm 3$ \\
\hline SR 9 & 140 & 81.8 & $3.0 \pm 0.7$ \\
\hline AS 205 & 160 & 184.0 & $2 \pm 2$ \\
\hline
\end{tabular}

F. 3. The relative velocities of the components of $T$ Tauri binary stars plotted in (a) cartesian coordinates $\left(v_{d \hat{e} c}\right.$ vs $\left.v_{r a}\right)$ and (b) polar coordinates $\left(v_{\hat{r}}\right.$ vs $\left.v \hat{\theta}\right)$. The observed relative velocities are inconsistent with that expected from a T Tauri star and (1) Herbig-Haro object-too small and not purely radial and positive, i.e., along dashed line in (b), (2) a background object-too small, (3) another cloud member-too large compared to the velocity dispersion of stars in these star forming regions (shown as dotted circles).

tween the average instantaneous separation, $\langle a\rangle$, and the semimajor axis. We modified this prescription in two ways: (1) the eccentricity is not fixed to zero, but allowed to vary and the probability of observing a specific eccentricity is given by $p(e)=2 e$, which was measured by Duquennoy \& Mayor (1991) for binary stars with periods longer than 1,000 days and (2) the relationship between the average instantaneous velocity, $\langle v\rangle$, and the velocity expected for a circular face-on orbit with the same semimajor axis and mass, $v_{\text {face-on circular }}$, is also calculated. These simulations resulted in the following relationships:

$$
\langle a\rangle=0.91 A,
$$

The comparison curves, based on these relationships, are shown in Fig. 4 for the cases of $\mathscr{C l}_{\text {tot }}$ equal to $0.2,1.0,3$, and 7. $\mathscr{C}_{0}$. Although the individual masses are untrustworthy, since the instantaneous velocities and separations measured can be quite different than the averages, the median value of 1.7. $\mathscr{C}_{0}$ should be representative of the true average total mass. Uncertainties in the distances to the star-forming regions studied (Kenyon et al. 1994; de Gues et al. 1989) should introduce only a $\sim 20 \%$ uncertainty into this statistical determination of the average total mass of these binary star systems. The median value of $1.7 \mathscr{C}_{0}$ for the sum of the two components' masses supports the theory that the individual $\mathrm{T}$ Tauri stars in these binary stars systems have masses lower than $3 \mathscr{C}_{0}$ from a dynamical point of view. However a larger sample is needed to analyze the distribution of total 


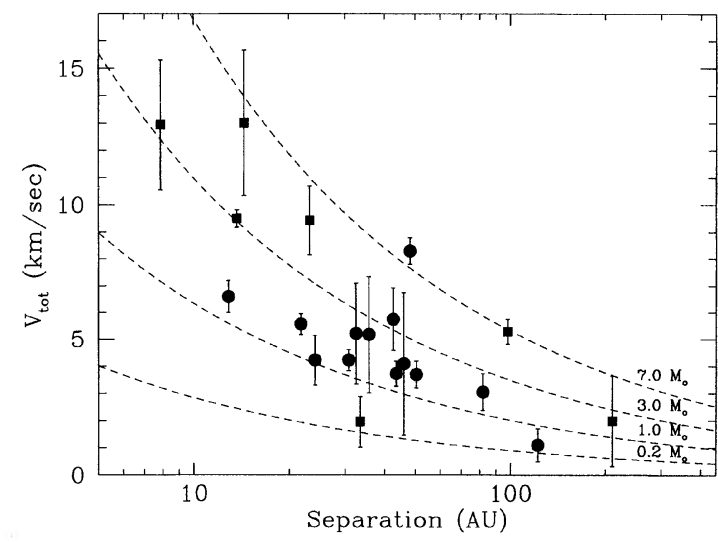

FIG. 4. The relative velocity of the binary stars' components as a function of their mean separation. The observed velocities are consistent with orbital motion as they (1) decrease with separation and (2) are generally greater for systems with higher mass primary stars $\left(\mathscr{A b}_{1}>1 \mathscr{A}_{0}\right.$ and $\mathscr{A b}_{1} \leqslant 1 \mathscr{L}_{0}$ are plotted as squares and circles, respectively). The measurements are compared to that expected from randomly observed binary stars with total masses of $0.2,1,3$, and $7 \mathscr{M}_{0}$. The sample has an average total dynamical mass of $\sim 1.7 \mathscr{C}_{0}$, although it should be cautioned that any individual total mass estimate is unreliable as discussed in the text.

masses in any detail. To obtain direct orbital element solutions and consequently mass estimates, which are independent of theoretical evolutionary tracks, the separation measurements have to deviate significantly from a straight line. With the current uncertainties of a few milliarcseconds and continued monitoring, solutions for binary stars separated by $\sim 0$. 1 should be obtainable in the next $\sim 5$ years.

\subsection{Effects of Additional Companion Stars}

Six of the sources included in this program are known to have additional companion stars, which are not detected by speckle imaging either because they are too widely or closely separated. Since each of these systems is hierarchical, i.e., the ratio of separations in the systems is large $(\geqslant 10)$, their orbits are expected to be stable and describable by the orbit of the close binary star pair superimposed on that of the wider pair $\left(P_{\text {close }}<P_{\text {wide }}\right)$. The perturbation from additional companions that are separated by larger distances than the pairs studied (outside the field of view), such as in the case of GG Tau and UZ Tau, are below our detection limit and thus do not effect the reported relative motions. The unresolved third components that were identified by Mathieu et al.'s (1989) spectroscopic work in 155913-2233 and ROX $42 \mathrm{C}$ have orbital periods of 2 and 36 days, respectively. A close companion has also been recently found in V773 Tau

TABLE 4. Average velocities for subsets of sample.

\begin{tabular}{l|cr}
\hline \hline & $\mathrm{M}_{1}<1 \mathrm{M}_{\mathrm{O}}$ & \multicolumn{1}{c}{$\mathrm{M}_{1}>1 \mathrm{M}_{0}$} \\
\hline Separation < 35 AU & $5.5 \mathrm{~km} / \mathrm{sec}(5)$ & $11.3 \mathrm{~km} / \mathrm{sec} \mathrm{(5)}$ \\
Separation > 35 AU & $3.4 \mathrm{~km} / \mathrm{sec}$ (7) & $3.1 \mathrm{~km} / \mathrm{sec} \mathrm{(2)}$ \\
\hline \hline
\end{tabular}

The number of binary stars in each set is given in parentheses. with an orbital period of 51 days (Welty 1995). If the close pairs have equal mass (and $K$ flux density) components, the observed motions of the wider pairs are not expected to be perturbed by more than 1 mas, which would not be detectable by the measurements reported here. However in the case of V807 Tau, the close pair, discovered with lunar occultation (Simon et al. 1995), is separated by $\sim 23$ mas. Thus the perturbation of the wider pair's orbit that was measured could be as much as the semimajor axis of the closer pairs photocentric orbit $(\alpha)$. If for the sake of argument the close pair has $A=3 \mathrm{AU}(23 \mathrm{mas})$ and a total mass of $1 \mathscr{C l}_{0}$, the orbital period would be 5 years. This "perturbation" would in fact dominate the relative velocity measured over the course of this study. We believe that the measured motion in V807 Tau comes from such an effect and note that in the future additional companion stars could be found by closely monitoring the separation of known binary stars.

\section{CONCLUSIONS}

We have embarked upon a program of monitoring the separations of $\mathrm{T}$ Tauri binary stars reported in Ghez et al. (1993) with two-dimensional near-infrared speckle imaging. The aim of this project is to investigate whether the observed companions are (1) stellar and (2) gravitationally bound and hence perturbing their circumstellar environment.

From each observation a binary star's separation is typically measurable to within a few milliarcseconds. With this high level of accuracy, we are on average sensitive to relative velocities of $\geqslant 5$ mas/yr $(3 \sigma)$ over the 5 year time baseline of this study (1989 December to 1994 December). This limiting velocity can be improved in the future by (1) increasing the time baseline of the observations (i.e., continuing this program) and (2) decreasing the pixel scale uncertainties by observing more calibration binary stars. At present, 17 of the 20 program $\mathrm{T}$ Tauri binary stars have undergone significant relative motion. On the basis of these relative velocity measurements it is possible on the one hand to rule out the suggestions that the secondary stars are (1) Herbig-Haro objects, (2) background sources, and (3) widely separated cloud members lying along the line of sight. The relative motion of the components depends on both the average separation and the primary star mass, which leads us to the conclusion that the observed separation changes are the result of orbital motion. This is the first demonstration that these systems are gravitationally bound. A comparison of the measured relative velocities with the average expected for randomly observed binary stars demonstrates that the average total mass of these systems is $\sim 1.7 \mathscr{O}_{0}$.

Although none of the binary star pairs included in this study have traversed enough of their orbit over the course of this study to provide useful orbital element solutions, a few are moving rapidly enough that useful solutions should be obtainable within the next 5 years (SR20, DF Tau, and V773 Tau). As demonstrated by V807 Tau, these precise separation measurements can potentially reveal the presence of additional (third) companion stars that are too close to be spatially resolved with speckle imaging but can influence the measured separation between the wider pair of stars. With 
TABLE 5. Measurements of calibration binary stars.

\begin{tabular}{|c|c|c|c|c|c|c|c|c|}
\hline \multicolumn{9}{|c|}{ CIT-1a Measurements } \\
\hline \multirow[t]{2}{*}{ ADS } & \multirow[t]{2}{*}{ Name } & \multirow{2}{*}{$\begin{array}{l}\text { Date } \\
(U T)\end{array}$} & \multicolumn{2}{|c|}{ Prediction } & \multicolumn{2}{|c|}{ Measured } & \multirow{2}{*}{$\begin{array}{c}\text { Scale } \\
\text { (mas/pix) }\end{array}$} & \multirow{2}{*}{$\begin{array}{l}\text { Orientation } \\
\text { (deg) }\end{array}$} \\
\hline & & & $\begin{array}{c}\text { Separation } \\
\text { (arcsec) }\end{array}$ & $\begin{array}{c}\text { PA } \\
(d e g)\end{array}$ & $\begin{array}{c}\text { Separation } \\
\text { (pixel) }\end{array}$ & $\begin{array}{c}\text { PA } \\
(\text { deg })\end{array}$ & & \\
\hline 755 & 36 And & 1990 Nov 11 & 0.7663 & 294.48 & $13.71 \pm 0.01$ & $288.94 \pm 0.08$ & 55.9 & -5.5 \\
\hline \multirow[t]{2}{*}{940} & $\phi$ And & 1990 Oct 04 & 0.4661 & 132.78 & $9.10 \pm 0.02$ & $131.7 \pm 0.1$ & 51.2 & -1.1 \\
\hline & & 1990 Nov 11 & 0.4661 & 132.78 & $9.10 \pm 0.02$ & $131.7 \pm 0.1$ & 51.2 & -1.0 \\
\hline \multirow[t]{2}{*}{4241} & $\sigma$ Ori & 1989 Dec 08 & 0.2344 & 137.10 & $4.67 \pm 0.02$ & $136.3 \pm 0.2$ & 50.2 & -0.8 \\
\hline & & 1990 Oct 05 & 0.2349 & 135.23 & $4.750 \pm 0.006$ & $134.0 \pm 0.4$ & 49.5 & -1.2 \\
\hline 7390 & $\omega$ Leo & $1989 \operatorname{Dec} 10$ & 0.4588 & 52.59 & $8.55 \pm 0.03$ & $54.2 \pm 0.3$ & 53.7 & 1.6 \\
\hline 11260 & SAO 103612 & 1990 Jul 08 & 0.3350 & 97.26 & $7.12 \pm 0.04$ & $106.0 \pm 0.2$ & 47.1 & 8.7 \\
\hline 11479 & SAO 86224 & 1992 July 26 & 0.6726 & 7.23 & $12.79 \pm 0.01$ & $7.73 \pm 0.08$ & 52.6 & 0.5 \\
\hline 14073 & $\beta$ Del & 1991 May 05 & 0.2805 & 199.69 & $4.75 \pm 0.01$ & $199.9 \pm 0.2$ & 59.1 & 0.2 \\
\hline \multirow[t]{3}{*}{14787} & $\tau \mathrm{Cyg}$ & 1990 Oct 03 & 0.5714 & 15.03 & $11.62 \pm 0.02$ & $10.41 \pm 0.03$ & 49.2 & -4.6 \\
\hline & & 1990 Oct 05 & 0.5717 & 14.97 & $11.62 \pm 0.02$ & $10.5 \pm 0.1$ & 49.2 & -4.5 \\
\hline & & 1991 Nov 21 & 0.6269 & 3.10 & $12.64 \pm 0.01$ & $359.6 \pm 0.1$ & 49.6 & -3.5 \\
\hline 14839 & SAO 107015 & 1990 Oct 04 & 0.2664 & 263.69 & $2.98 \pm 0.03$ & $271.6 \pm 0.5$ & 89.4 & 7.9 \\
\hline 16314 & HO 482 & 1990 Oct 03 & 0.4017 & 27.98 & $7.43 \pm 0.02$ & $29.8 \pm 0.2$ & 54.1 & 1.8 \\
\hline 16345 & BU 382 & 1990 Oct 03 & 1.0245 & 216.53 & $17.58 \pm 0.02$ & $215.73 \pm 0.02$ & 58.3 & -0.8 \\
\hline 16836 & 72 Peg & 1990 Nov 11 & 0.5271 & 89.74 & $9.78 \pm 0.02$ & $87.68 \pm 0.02$ & 53.9 & -2.1 \\
\hline
\end{tabular}

CIT-1b Measurements

\begin{tabular}{clllrrrrr}
\hline \hline 940 & $\phi$ And & 1993 Nov 25 & 0.4672 & 130.69 & $12.9 \pm 0.3$ & $130.0 \pm 0.3$ & 36.2 & -0.7 \\
- & SAO 42642 & 1993 Nov 27 & 0.4867 & 178.70 & $12.58 \pm 0.04$ & $177.3 \pm 0.2$ & 38.7 & -1.4 \\
7390 & $\omega$ Leo & 1993 Nov 27 & 0.4917 & 66.66 & $12.51 \pm 0.06$ & $68.1 \pm 0.1$ & 39.3 & 1.4 \\
11479 & SAO 86224 & 1993 May 10 & 0.6758 & 7.09 & $17.70 \pm 0.04$ & $6.54 \pm 0.07$ & 38.2 & -0.6 \\
& & 1993 Nov 29 & 0.6781 & 6.99 & $17.59 \pm 0.07$ & $6.8 \pm 0.1$ & 38.6 & -0.2 \\
14073 & B Del & 1993 Nov 29 & 0.2002 & 84.99 & $4.9 \pm 0.1$ & $87 \pm 2$ & 40.9 & 2.0 \\
14787 & $\tau$ Cyg & 1993 Nov 26 & 0.7098 & 346.16 & $19.5 \pm 0.1$ & $342.8 \pm 0.3$ & 36.4 & -3.4 \\
16345 & BU 382 & 1993 Nov 26 & 1.0104 & 219.38 & $23.72 \pm 0.02$ & $217.48 \pm 0.07$ & 42.6 & -1.9 \\
16836 & 72 Peg & 1993 Nov 25 & 0.5298 & 92.28 & $13.52 \pm 0.03$ & $90.27 \pm 0.06$ & 39.2 & -2.0 \\
\hline \hline
\end{tabular}

CIT-2 Measurements

\begin{tabular}{rlrlrrrrr}
\hline \hline 755 & 36 And & 1994 Oct 21 & 0.8405 & 303.43 & $22.16 \pm 0.07$ & $297.3 \pm 0.2$ & 37.9 & -6.1 \\
940 & $\phi$ And & 1994 Oct 21 & 0.4674 & 130.11 & $13.65 \pm 0.06$ & $128 \pm 1$. & 34.2 & -2.0 \\
- & SAO 42642 & 1994 Dec 20 & 0.4309 & 156.40 & $11.59 \pm 0.05$ & $155.32 \pm 0.07$ & 37.2 & -1.1 \\
7390 & $\omega$ Leo & 1994 Dec 20 & 0.5035 & 70.07 & $14.32 \pm 0.01$ & $70.81 \pm 0.06$ & 35.2 & 0.7 \\
14787 & $\tau$ Cyg & 1994 Dec 21 & 0.7400 & 338.50 & $21.66 \pm 0.05$ & $336.4 \pm 0.1$ & 34.2 & -2.1 \\
16836 & 72 Peg & 1994 Oct 21 & 0.5304 & 93.00 & $15.03 \pm 0.01$ & $89.2 \pm 0.1$ & 35.3 & -3.8 \\
\hline
\end{tabular}


TABLE 5. (continued)

\begin{tabular}{|c|c|c|c|c|c|c|c|c|}
\hline \multicolumn{9}{|c|}{ CIT-2 Measurements } \\
\hline 16314 & HO 482 & $1994 \operatorname{Dec} 20$ & 0.4254 & 24.48 & $11.94 \pm 0.01$ & $27.13 \pm 0.04$ & 35.6 & 2.64 \\
\hline 16345 & BU 382 & $1994 \operatorname{Dec} 20$ & 1.0038 & 220.37 & $26.41 \pm 0.06$ & $219.51 \pm 0.04$ & 38.0 & -0.86 \\
\hline \multicolumn{9}{|c|}{ SO-1 Measurements } \\
\hline 61 & STF 3062 & 1994 Sep 22 & 1.4909 & 317.90 & $19.52 \pm 0.03$ & $325.3 \pm 0.2$ & 76.4 & 7.4 \\
\hline \multirow[t]{2}{*}{755} & 36 And & $1993 \operatorname{Dec} 26$ & 0.8253 & 301.75 & $10.50 \pm 0.03$ & $301.51 \pm 0.08$ & 78.6 & -0.2 \\
\hline & & 1994 Sep 22 & 0.8395 & 303.32 & $10.67 \pm 0.05$ & $302.4 \pm 0.1$ & 78.7 & -0.9 \\
\hline 940 & $\phi$ And & $1993 \operatorname{Dec} 24$ & 0.4672 & 130.64 & $6.51 \pm 0.09$ & $136 \pm 1$ & 71.8 & 5.4 \\
\hline \multirow[t]{2}{*}{-} & SAO 42642 & $1993 \operatorname{Dec} 26$ & 0.4827 & 177.21 & $6.92 \pm 0.08$ & $188 \pm 1$ & 69.8 & 10.8 \\
\hline & & 1994 Jan 29 & 0.4779 & 175.4 & $6.74 \pm 0.04$ & $181.3 \pm 0.2$ & 70.9 & 5.9 \\
\hline \multirow[t]{2}{*}{7390} & $\omega$ Leo & $1993 \mathrm{Dec} 26$ & 0.4925 & 66.92 & $7.34 \pm 0.04$ & $72.2 \pm 0.2$ & 67.1 & 5.3 \\
\hline & & 1994 Jan 29 & 0.4935 & 67.2 & $7.29 \pm 0.01$ & $68.6 \pm 0.2$ & 67.7 & 1.4 \\
\hline 11046 & $70 \mathrm{Oph}$ & 1994 Sep 22 & 2.3888 & 169.86 & $32.44 \pm 0.04$ & $181.17 \pm 0.08$ & 73.6 & 11.3 \\
\hline 11077 & $99 \mathrm{Her}$ & 1994 Sep 22 & 0.5366 & 53.67 & $9.8 \pm 0.1$ & $48.2 \pm 0.5$ & 54.5 & -5.5 \\
\hline \multirow[t]{3}{*}{11479} & SAO 86224 & 1992 Oct 11 & 0.6734 & 7.19 & $10.06 \pm 0.04$ & $18.4 \pm 0.2$ & 66.9 & 11.2 \\
\hline & & 1994 Sep 22 & 0.6813 & 6.85 & $10.12 \pm 0.03$ & $17.4 \pm 0.2$ & 67.3 & 10.6 \\
\hline & & 1994 Sep 23 & 0.6813 & 6.84 & $10.14 \pm 0.02$ & $17.3 \pm 0.1$ & 67.2 & 10.5 \\
\hline 11871 & BU 648 & 1994 Sep 22 & 0.7786 & 358.31 & $10.86 \pm 0.04$ & $7.9 \pm 1$ & 71.7 & 9.6 \\
\hline \multirow[t]{2}{*}{14787} & $\tau$ Cyg & 1992 Oct 10 & 0.6669 & 355.13 & $10.2 \pm 0.1$ & $3.1 \pm 0.3$ & 65.4 & 8.0 \\
\hline & & 1994 Sep 22 & 0.7340 & 340.21 & $10.61 \pm 0.03$ & $347.38 \pm 0.07$ & 69.2 & 7.2 \\
\hline 15972 & KR 60 & 1994 Sep 22 & 3.3145 & 116.23 & $44.9 \pm 0.9$ & $30 . \pm 1$ & 73.8 & 3.8 \\
\hline 16314 & HO 882 & 1994 Sep 23 & 0.4241 & 24.67 & $6.38 \pm 0.03$ & $37 \pm 1$ & 66.5 & 12.1 \\
\hline 16345 & BU 382 & 1994 Sep 23 & 1.0054 & 220.16 & $14.33 \pm 0.03$ & 225.9 & 70.2 & 5.8 \\
\hline \multirow[t]{2}{*}{16836} & 72 Peg & $1993 \operatorname{Dec} 26$ & 0.5298 & 92.34 & $7.48 \pm 0.02$ & $92.67 \pm 0.04$ & 70.8 & 0.3 \\
\hline & & 1994 Sep 22 & 0.5304 & 92.96 & $7.59 \pm 0.02$ & $92.44 \pm 0.09$ & 69.9 & -0.5 \\
\hline
\end{tabular}

this technique and the present pixel scale uncertainties, one can reliably find additional pairs with $\alpha \geqslant 15$ mas (2AU), where $\alpha$ is the semimajor axis of the close pair's photocentric orbit.

Given the high frequency of companions observed at separations less than $100 \mathrm{AU}$ and the present evidence that the companion stars are gravitationally bound, it is clear that the effects of these secondary stars should not be overlooked in modeling the circumstellar disk environment of these stars.

We thank the staff of the Steward $90^{\prime \prime}$ and Palomar Observatories, especially the night assistants Juan Carrasco, Dennis Means, and Vic Hansen, and an anonymous referee for helpful comments. A.G. receives support from NASA through Grant No. HF-1031.01-92A awarded by the Space Telescope Science Institute which is operated by the Asso- ciation of Universities for Research in Astronomy, Inc., for NASA under Contract No. NAS5-26555. D.W.M. is supported by NSF under Grant No. AST92-03336. Infrared astronomy at Caltech is supported by a grant from NSF and A.W. is supported through the NASA Graduate Student Researchers Program.

\section{APPENDIX: CALIBRATION BINARY STARS}

Observations of binary stars with well known orbital elements, "calibration binary stars," serve two purposes: (1) they test the reproducibility of the binary star separation measurements and (2) they measure the pixel scale and orientation of the detectors. The calibration binary stars were observed and analyzed with the same techniques used to analyze the T Tauri binary stars discussed in Secs. 2 and 3. In 

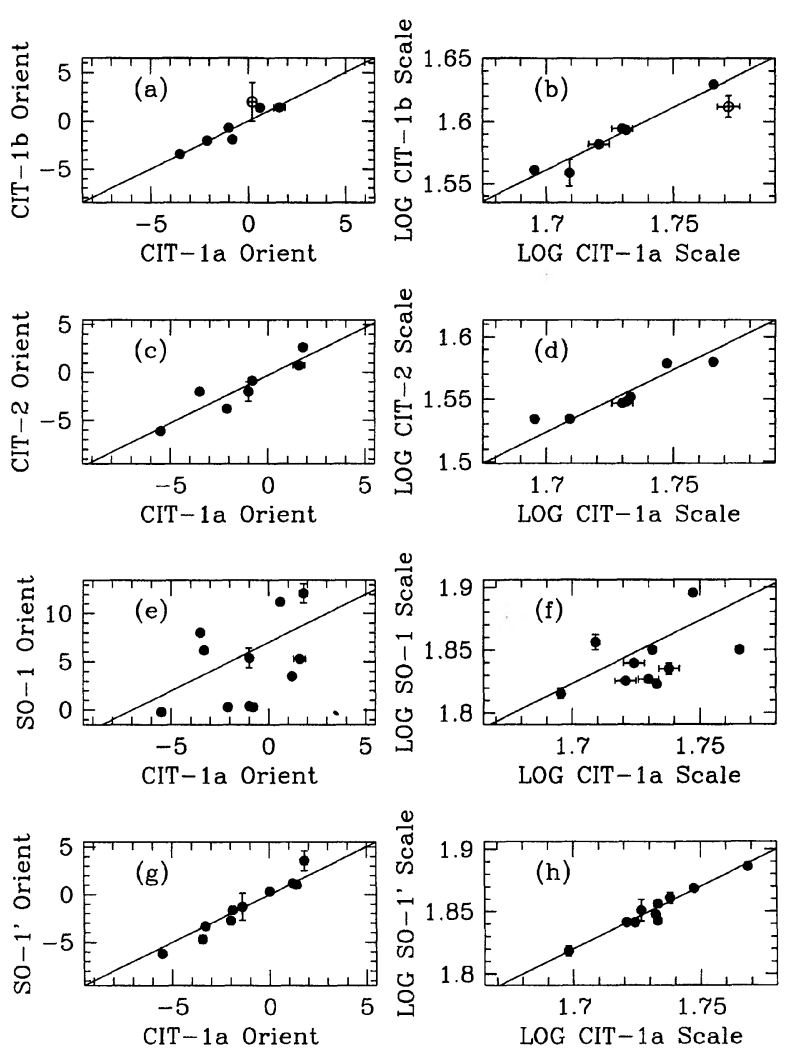

FIG. 5. The estimates of the pixel scale and orientation from the calibration binary stars of CIT-1b (a,b), CIT-2 (c,d), and SO-1 (e,f) with respect to CIT-1a. The pixel scales are in units of mas/yr and the orientations are in degrees. The uncertainties plotted are based on the standard deviations of the binary star separation and position angle that were obtained from fits to the individual obj/ref pairs, as described in Sec. 2. The relative pixel scales and orientations are derived from the linear fits to these points. SO-1 has a characterizable field distortion that causes the pixel scale and orientation to depend on the orientation of the observed binary stars (see Table 1). Once this correction term is removed (SO-1'; g,h) the relative pixel scale and orientation an be determined to accuracies similar to the other systems used.

Table 5 we provide a compilation of the calibration binary results, which includes (1) the dates of the observations, (2) the predicted separations (based on the orbital elements from Worely \& Heinz 1983), (3) the measured separations in pixels and position angles in degrees with respect to the detector axes, and (4) the resulting pixel scale and detector orientation estimates. Repeated observations of a number of these calibration binary stars over short time periods $(t \ll$ orbital period; such that one does not expect the separations to have undergone measurable changes) demonstrate that for each camera the measurements are reproducible since the variations $(<1 \%)$ in the measured binary star separations are less than the quoted uncertainties, which were obtained from the standard deviations of the individual obj/ref pair results (see Sec. 2).

The average pixel scale and orientation based on the calibration binary stars are listed in columns 3 and 4 of Table 1 for each camera. The scatter in these estimates are caused by uncertainties in the orbital elements as well as any optical aberrations the cameras may have. It should be noted that one star, ADS 14839 (measured only by CIT-1a), is omitted from the average based on the student $t$ test. On the basis of these measurements, the absolute pixel scales and orientations can be determined with a precision of $2 \%-3 \%$. Observations of the same calibration binary stars with two different cameras (1) measure the relative pixel scales and orientations and (2) reveal any relative optical distortion in the cameras. The advantage of these relative quantities is that they are unaffected by uncertainties in the orbital elements, because the time lags between the measurements are short compared to the orbital periods. Furthermore, for this project of detecting orbital motion of $\mathrm{T}$ Tauri binary stars it is crucial to intercalibrate the cameras as accurately as possible. Since CIT-1a measured the largest number of T Tauri binary stars, the relative pixel scales and orientations are stated with respect to this camera. Figure 5 plots the pixel scale and orientation of each camera against that for CIT-1a as determined by calibration binary stars that were measured by both cameras; in other words, each point represents two measurements of one calibration binary star. If the orbital elements were perfectly known, all the points would be in one location. Errors in the orbital elements should spread these points out along a $45^{\circ}$ line, which can be fit to determine the relative pixel scale/orientation between the cameras. The unfilled points in Fig. 5 represent one binary star for which the predicted vector separation changed by $30 \%$ between the two measurements, compared to the rest which have changed by at most $10 \%$, and thus uncertainties in the orbital elements have a significant effect. The relative pixel scales and orientations of CIT-1b and CIT-2 with respect to CIT-1a are determined by a linear fits to within $\sim \pm 0.5 \%$. However in the case of SO-1 with respect to CIT-1a, the scatter around a line is much greater than can be explained by the fitting uncertainties or uncertainties in the orbital elements. The additional source of noise comes from a slight field distortion in SO-1, which is easily characterized from these measurements [see Table 1 and Figs. 5(g) and 5(h)]. Once this distortion is corrected for the pixel scale and orientation of SO-1 with respect to CIT-1a is measurable to within $1 \%$ uncertainties. The absolute scales/orientations for CIT-1b, CIT-2, and SO-1 used in Table 1 are determined by multiplying the relative scales/orientations with the absolute scale for CIT-1a.

\section{REFERENCES}

Adams, F. C., Ruden, S., \& Shu, F. H. 1989, ApJ, 347, 959

Aitken, R. G. 1932, in A New General Catalog of Double Stars, Carnegie

Institution of Washington Publication No. 417

Bertian, F. C. 1958, ApJ, 128, 533

Bonnell, I. 1994, MNRAS, 269, 837

Bonnell, I., \& Bastien, P. 1992, ApJ, 401, L31
Bonnell, I., \& Bastien, P. 1993, ApJ, 406, 614

Boss, A. P. 1993, ApJ, 410, 157

de Geus, E. J., \& Burton, W. B. 1991, A\&A, 246, 559

de Geus, E. J., de Zeeuw, P. T., \& Lub, J. 1989, A\&A, 216, 44

Duquennoy, A., \& Mayor, M. 1991, 248, 485

Dutrey, A., Guilloteau, S., \& Simon, M. 1994, A\&A, 286, 149 
Edwards, S., Ray, T., \& Mundt, R. 1993, in Protostars and Planets III, edited by E. H. Levy and J. I. Lunine, p. 567

Fischer, D. A., \& Marcy, G. W. 1992, 396, 178

Ghez, A. M. 1992, Ph.D. thesis, California Institute of Technology

Ghez, A. M., Emerson, J. P. Graham, J. R., Meixner, M., \& Skinner, C. 1994, ApJ, 434, 707

Ghez, A. M., Neugebauer, G., \& Matthews, K. 1993, AJ, 106, 2005

Heintz, W. D. 1980, ApJS, 44, 111

Jensen, E. L. N., Mathieu, R. D., \& Fuller, G. A. 1994, ApJ, 429, L29

Jones, B. F., \& Herbig, G. H. 1979, AJ, 84, 1872

Kenyon, S. J., Dobrzycka, D., \& Hartmann, L. 1994, AJ, 108, 1872

Koresko, C. D, Beckwith, S. V. W., Chez, A. M., Matthews, K., \& Neugebauer, G. 1991, AJ, 102, 2073

Leinert, Ch., Zinnecker, H., Weitzel, N., Christou, J., Ridgway, S. T., Jameson, R., Haas, M., \& Lenzen, R. 1993, A\&A, 278, 129

Magazzu, A., \& Martin, E. L. 1994, A\&A, 287, 571

Mathieu, R. D., Adams, F. C., \& Latham, D. W. 1991, AJ, 101, 2184

Mathieu, R. D., Walter, F. M., Myers, P. C. 1989, AJ, 98, 987
McCarthy, Jr., D. W., Henry, T. J., McLeod, B., \& Christou, J. C. 1991, AJ, 101,214

McCarthy, Jr., D. W., McLeod, B. A., \& Barlow, D. 1990, SPIE, 1237, 496 McDonald, J. M., \& Clarke, C. J. 1993, MNRAS, 262, 800

McDonald, J. M., \& Clarke, C. J. 1995, MNRAS (in press)

Osterloh, M., \& Beckwith, S. V. W. 1995, ApJ, 439, 288

Ostriker, E. C., Shu, F. H., \& Adams, F. 1992, ApJ, 428

Petrie, R. M. 1961, MNRAS, 123, 501

Schwartz, R. D. 1983, ARA\&A, 21, 209

Schwartz, R. D. 1986, Can. J. Phys., 64, 414

Simon, M., Chen, W. P., Howell, R. R., Benson, J. A., \& Slowik, D. 1992 , ApJ, 384, 212

Simon, M., Ghez, A. M., Leinert, Ch. 1993, ApJ, 408, L33

Simon, M., et al. 1995, ApJ, 443, 625

Terquem, C., \& Bertourt, C. 1993, A\&A, 274, 291

Welty, A. D. 1995, AJ (in press)

Worley, C. E., \& Heintz, W. D. 1983, PUSNO, 24, part 7 